\title{
Wilson chiral perturbation theory, Wilson-Dirac operator eigenvalues and clover improvement
}

\section{Poul H. Damgaard}

Niels Bohr International Academy and Discovery Center, Niels Bohr Institute

University of Copenhagen

Blegdamsvej 17, DK-2100 Copenhagen $\emptyset$, Denmark

\section{Urs M. Heller*}

American Physical Society, One Research Road, Ridge, NY 11961, USA

E-mail: hellerdaps.org

\section{Kim Splittorff}

Discovery Center, Niels Bohr Institute, University of Copenhagen

Blegdamsvej 17, DK-2100 Copenhagen $\emptyset$, Denmark

Chiral perturbation theory for eigenvalue distributions, and equivalently random matrix theory, has recently been extended to include lattice effects for Wilson fermions. We test the predictions by comparison to eigenvalue distributions of the Hermitian Wilson-Dirac operator from pure gauge (quenched) ensembles. We show that the lattice effects are diminished when using clover improvement for the Dirac operator. We demonstrate that the leading Wilson low-energy constants associated with Wilson (clover) fermions can be determined using spectral information of the respective Dirac operator at finite volume.

Xth Quark Confinement and the Hadron Spectrum

October 8 - 12, 2012

TUM Campus Garching, Munich, Germany

\footnotetext{
* Speaker.
} 


\section{Introduction}

The low-energy behavior of QCD, the spontaneous breaking of chiral symmetry, including the explicit breaking by the quark masses, is described by chiral perturbation theory $(\chi \mathrm{PT})$. In the lattice regularization of QCD lattice artifacts can contribute to the breaking of chiral symmetry, for Wilson fermions, or its partial breaking, in the case of staggered fermions. These effects can be included in the $\chi \mathrm{PT}$ approach, leading to new, lattice discretization dependent low-energy constants. We consider Wilson fermions in this contribution, for which the effective theory, Wilson $\chi \mathrm{PT}(\mathrm{W} \chi \mathrm{PT})$ was introduced and worked out in [1]. The new terms in the chiral Lagrangian affect the low-lying spectrum of the (Hermitian) Wilson-Dirac operator [⿰]. For a recent review with additional references, see Ref. [3]. Here, we test and verify the predictions for the distribution of the low-lying eigenvalues with lattice QCD simulations [ [ 4 , 5] and show [6] that they can be used to obtain the new low-energy constants introduced in W $\chi$ PT. We also demonstrate the effect of clover improving the Wilson-Dirac operator.

\section{The W $\chi$ PT and Wilson RMT framework}

We will be concerned with the $\varepsilon$-regime of $\mathrm{W} \chi \mathrm{PT}$ where the zero momentum modes dominate - the system size is such that $m_{\pi} L \ll 1$. In addition we adopt the power counting with $m \sim a^{2}$. Hence, dropping the kinetic part of the chiral Lagrangian, we consider

$$
\mathscr{L}=-\frac{1}{2} m \Sigma \operatorname{Tr}\left(U+U^{\dagger}\right)-\frac{1}{2} z \Sigma \operatorname{Tr}\left(U-U^{\dagger}\right)+a^{2} \mathscr{V} .
$$

The second term, representing a $\bar{\psi} \gamma_{5} \psi$ term, is introduced for later convenience. $\mathscr{V}$ describes the lattice artifacts [1]

$$
\mathscr{V}=W_{8} \operatorname{Tr}\left(U^{2}+U^{\dagger 2}\right)+W_{6}\left[\operatorname{Tr}\left(U+U^{\dagger}\right)\right]^{2}+W_{7}\left[\operatorname{Tr}\left(U-U^{\dagger}\right)\right]^{2} .
$$

At large $N_{c}$, the two-trace terms are suppressed.

The finite size scaling considered is such that

$$
\hat{m}=m \Sigma V, \quad \hat{z}=z \Sigma V \quad \text { and } \quad \hat{a}_{j}^{2}=a^{2} W_{j} V \quad \text { for } \quad j=6,7,8
$$

are held fixed. Here $\Sigma$ is the condensate and $V$ the volume.

This leading order in $\mathrm{W} \chi \mathrm{PT}$ can equivalently be described by a chiral random matrix theory (RMT). For Wilson fermions, including the one-trace term with low-energy constant $W_{8}$, the Dirac operator is represented in Wilson RMT (WRMT) as [2]

$$
\mathscr{D}_{W}=\left(\begin{array}{cc}
\tilde{a} A & i W \\
i W^{\dagger} & \tilde{a} B
\end{array}\right),
$$

with $W$ a random $(n+v) \times n$ complex matrix, and $A$ and $B$ random Hermitian matrices of size $(n+v) \times(n+v)$ and $n \times n$, respectively. As usual in the RMT context, we consider a fixed index $v$. We use a chiral basis with $\gamma_{5}=\operatorname{diag}(1, \ldots, 1,-1, \ldots,-1)$. $A$ and $B$ represent the chiral symmetry breaking term corresponding to the Wilson term in the Wilson-Dirac operator. 


\begin{tabular}{|c|c|c|c|c|c|c|}
\hline Ens & $\beta_{I w}$ & $r_{0} / a$ & $a[\mathrm{fm}]$ & size & $L[\mathrm{fm}]$ & $|Q|=0,1,2$ cfgs \\
\hline A & 2.635 & 5.37 & 0.093 & $16^{4}$ & 1.5 & $1279,2257,1530$ \\
B & 2.635 & 5.37 & 0.093 & $20^{4}$ & 1.9 & $401,682,644$ \\
C & 2.79 & 6.70 & 0.075 & $20^{4}$ & 1.5 & $1207,2130,1448$ \\
\hline
\end{tabular}

Table 1: The ensembles used. The scale is set by $r_{0}=0.5 \mathrm{fm}$. The $r_{0} / a$ values come from interpolation formulae in [10]. $Q$ is the topological charge (see text).

The two-trace terms can be incorporated in WRMT via two Gaussian integrations

$$
Z^{v}\left(\hat{m}, \hat{z} ; \hat{a}_{6}, \hat{a}_{7}, \hat{a}_{8}\right)=\frac{1}{16 \pi \hat{a}_{6} \hat{a}_{7}} \int_{-\infty}^{\infty} d y_{6} d y_{7} \mathrm{e}^{-\frac{y_{6}^{2}}{16 \hat{a}_{6}^{2}}-\frac{\mathrm{y}_{7}^{2}}{16 \hat{a}_{7}^{2}}} \mathrm{Z}^{v}\left(\hat{\mathrm{m}}-\mathrm{y}_{6}, \hat{\mathrm{z}}-\mathrm{y}_{7} ; 0,0, \hat{a}_{8}\right) .
$$

Here,

$$
Z^{v}\left(\hat{m}, \hat{z} ; 0,0, \hat{a}_{8}\right)=\int d U \operatorname{det}^{v} U \mathrm{e}^{-\mathrm{V} \mathscr{L}\left(\mathrm{W}_{6}=\mathrm{W}_{7}=0\right)}
$$

is the fixed-index partition function with the one-trace $\mathscr{O}\left(a^{2}\right)$ term included.

\section{Index of the Wilson-Dirac operator}

As indicated above, RMT predictions apply to gauge field sectors with a fixed index, or, in the continuum, fixed topological charge. For the Wilson-Dirac operator, the index can be defined by

$$
v \equiv \sum_{k}^{\prime} \operatorname{sign}\left(\left\langle k\left|\gamma_{5}\right| k\right\rangle\right)
$$

with $|k\rangle$ the $k^{\prime}$ th eigenstate of the Wilson-Dirac operator, $D_{W}$. Only eigenvectors with real eigenvalues contribute, and the / indicates that only the real eigenvalues in the branch near zero, with eigenvalues $<r_{\text {cut }}$, are kept. Introducing the Hermitian Wilson-Dirac operator $D_{5}\left(m_{0}\right)=\gamma_{5}\left(D_{W}+m_{0}\right)$ and using

$$
D_{5}\left(m_{0}\right)|\psi\rangle=0 \quad \Rightarrow \quad D_{W}|\psi\rangle=-m_{0}|\psi\rangle
$$

the index can equivalently be obtained from the zero crossings of the spectral flow of $D_{5}\left(m_{0}\right)$ up to $m_{\text {cut }}=-r_{\text {cut }}$ [7]. It corresponds to the index of an overlap operator [8] with kernel $D_{5}\left(m_{\text {cut }}\right)$. Because of the dependence on the choice of $r_{\text {cut }}$, the index of the Wilson-Dirac operator is not unique.

\section{The numerical simulations}

For our numerical tests, in the quenched case, we generated three ensembles using the Iwasaki gauge action [9], which suppresses dislocations and gives a fairly unique index $v$ or topological charge $Q$. The ensembles are characterized in Table 1 .

The topological charge listed in Table 11 was obtained after six steps of HYP smearing [11] with an improved lattice $F \tilde{F}$ operator [12]. On the configurations with $|Q| \leq 1$, as well as the $|Q|=2$ configurations of ensemble A, we also did the much more expensive computation of the index 

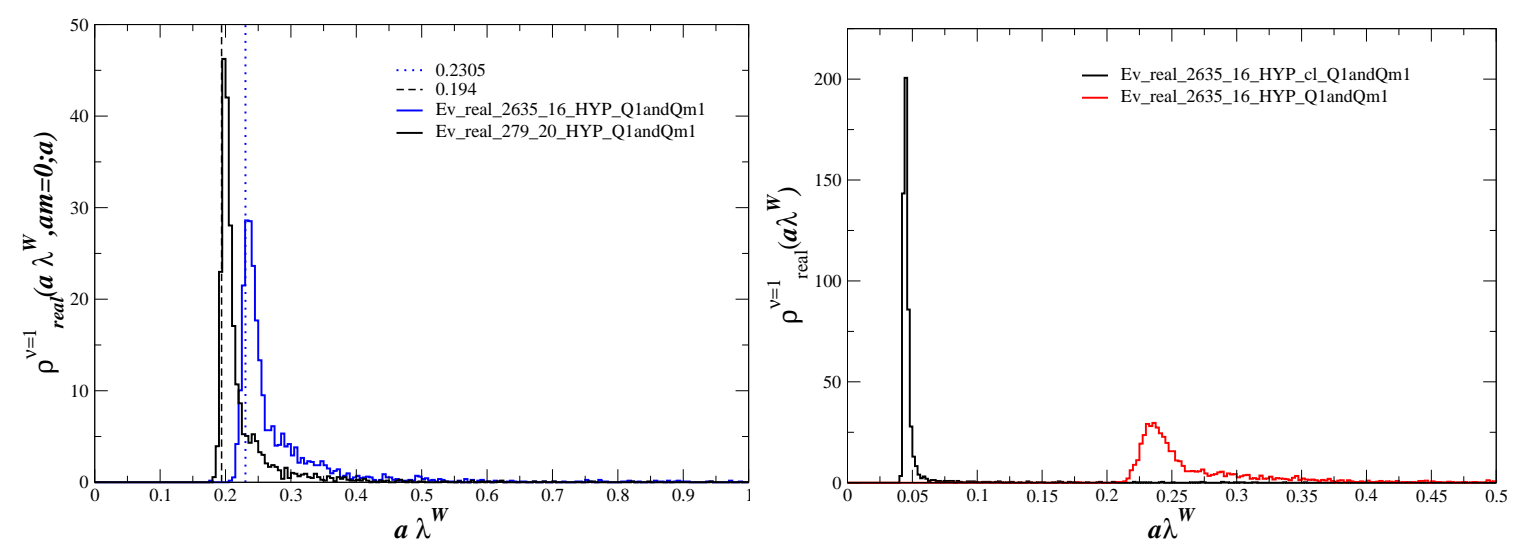

Figure 1: Distribution of the real eigenvalues of the Wilson-Dirac operator for the $v=1$ configurations of the two $L=1.5 \mathrm{fm}$ ensembles A and C (left) and comparison of the distribution with and without clover improvement at $a=0.093 \mathrm{fm}$ (ensemble A, right).

from the spectral flow. We first applied one HYP smearing before constructing the Wilson-Dirac operator. The topological charge and the index agreed on most configurations, with the agreement improving at smaller lattice spacing and becoming worse for the larger volume ensemble $\mathrm{C}$, for which it was about $97 \%$.

The crossing points in the spectral flow are the real eigenvalues, whose distribution is shown in Fig. 1 (left). The dashed vertical lines are estimates of (minus) the critical mass. Some real eigenvalues are smaller, on so-called "exceptional" configurations.

For ensemble A, we also computed the spectral flow with clover improving the Wilson-Dirac operator, again after one HYP smearing. The clover coefficient was set to 1, which is expected to be close to the nonperturbative value after the HYP smearing [13]. The resulting distribution of the real eigenvalues is compared to the unimproved case in Fig. 1 (right). The improvement is quite dramatic, besides the expected reduced shift away from zero, the distribution is much narrower and more symmetric. The width is determined by the $\mathscr{O}\left(a^{2}\right)$ terms in Eq. (2.2), so with clover improvement the coefficients are much smaller.

\section{Wilson eigenvalue distributions and WRMT}

We next computed the lowest 20, in magnitude, eigenvalues of the Hermitian Wilson-Dirac operator $D_{5}\left(m_{0}\right)$ with bare mass $a m_{0}=-0.216$ for ensembles A and B to compare to eigenvalues distributions obtained from WRMT [纤].

Without clover improvement, we considered only contributions from the two-trace term in Eq. (2.2) [4]. We used the $v=0$ histogrammed eigenvalue distributions of ensemble A to determine the WRMT parameters $\hat{m}$ and $\hat{a}=\hat{a_{8}}$ and the eigenvalue rescaling factor $\Sigma V$. Using the same parameters we then get a prediction for the $v=1$ distribution that can be compared to the numerical data (see Fig. 目 top).

Ensemble $\mathrm{B}$ differs from ensemble $\mathrm{A}$ only in the volume. Using volume scaling, $\hat{m}_{B}=$ $\hat{m}_{A}\left(V_{B} / V_{A}\right)$ and $\hat{a}_{B}=\hat{a}_{A} \sqrt{V_{B} / V_{A}}$, we obtain predictions for the distributions for ensemble $\mathrm{B}$ (see Fig. 2 bottom). As can be seen, the WRMT predictions work well. 

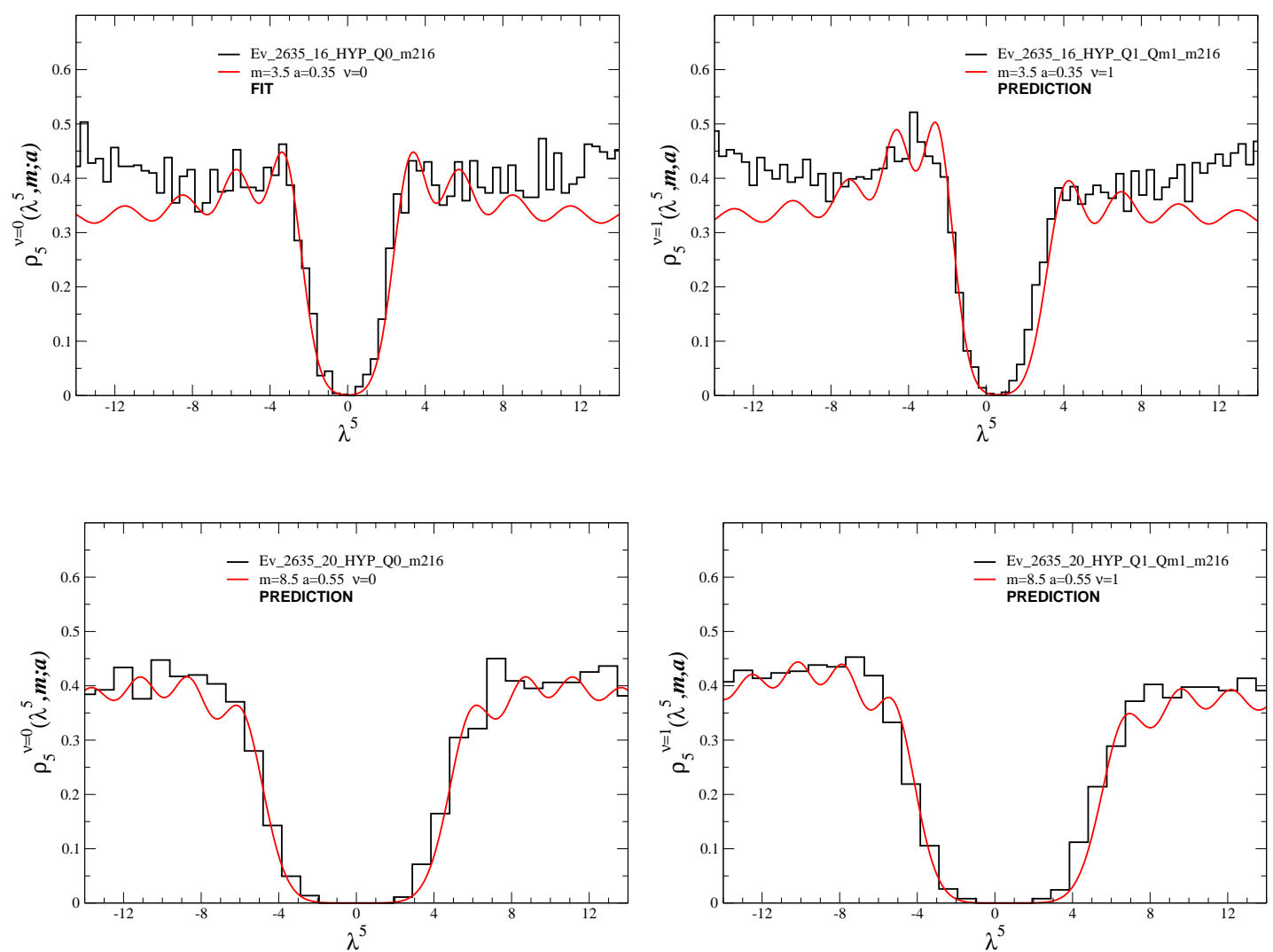

Figure 2: Comparison of the histogrammed eigenvalue distributions with WRMT. The $v=0$ distribution of ensemble A (top left) was used to obtain the parameters. The predictions for ensemble B (bottom) used volume scaling of the parameters.

For ensemble $\mathrm{C}$, at the smaller lattice spacing, we computed the eigenvalues with two different bare masses $a m_{0}=-0.178$ and -0.184 . We used the histogrammed $v=0$ distribution with bare mass $a m_{0}=-0.184$ to the determine the WRMT parameters, and used "mass scaling", $\Delta \hat{m}=$ $\Delta m_{0} \Sigma V$ for predictions for the distributions with the other bare mass $a m_{0}=-0.178$, as shown in Fig. 3. Again, the WRMT predictions work well.

\section{Clover improved eigenvalue distributions and WRMT}

We have already seen from the distribution of the real eigenvalues in Fig. 11(right) that clover improvement not only, as expected, decreases the additive mass renormalization (the real eigenvalue peak is much closer to zero) but also the size of the $\mathscr{O}\left(a^{2}\right)$ low-energy constants considerably (the distribution becomes much narrower). Here we consider the effects on the distribution of the 20 lowest, in magnitude, eigenvalues of the Hermitian Wilson-Dirac operator $D_{5}\left(m_{0}\right)$ with clover improvement for ensemble A using a bare mass $a m_{0}=-0.03$. The comparison with WRMT is shown in the first three panels of Fig. A.

For $\left|\hat{a}_{j}\right| \ll 1$ the lattice effects affect, to leading order, only the index peak of the topological modes. These are the lowest eigenvalues with almost chiral eigenvectors which correspond to the real eigenvalues shifted by the bare mass. As can be seen in Fig. $\bigoplus$ (bottom right) the distributions 

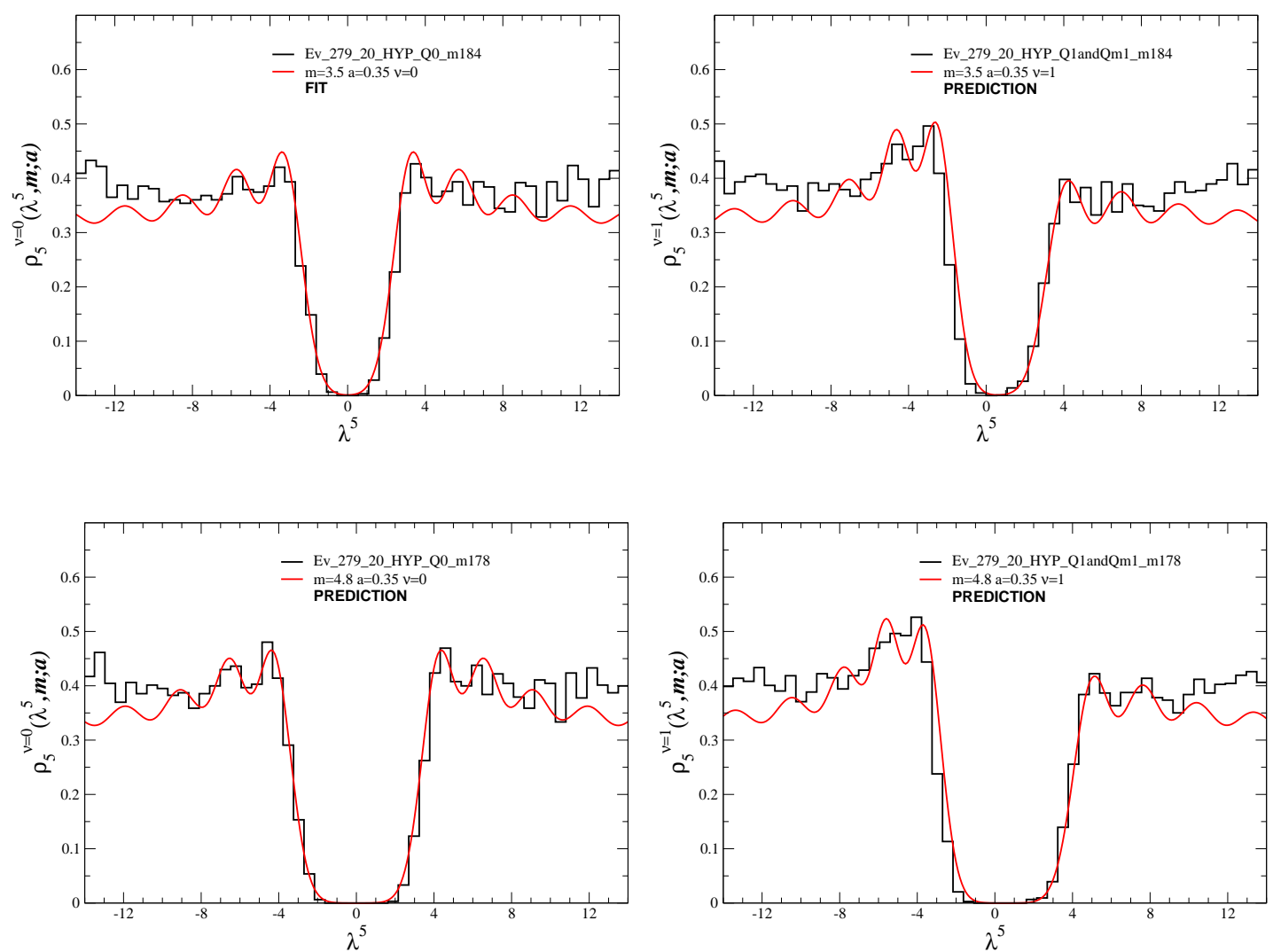

Figure 3: Comparison of the histogrammed eigenvalue distributions with WRMT for ensemble C. The $v=0$ distribution with bare mass $a m_{0}=-0.184$ (top left) was used to obtain the parameters. The predictions for bare mass $a m_{0}=-0.178$ (bottom) used "mass scaling", $\Delta \hat{m}=\Delta m_{0} \Sigma V$.

match almost perfectly. The eigenvalue density, for $|v|>0$, on the opposite side of the index peak is almost continuum like and allows determination of $\hat{m}$ and $\Sigma V$. We use the $|v|=1$ eigenvalue distribution for this. The $\hat{a}_{j}$ are then obtained from their effect on the index peak. We use the fact that the low-energy constant have fixed signs $W_{6}<0, W_{7}<0$ and $W_{8}>0$ [2, 14, 15] and that the distribution depends only on the combination $\left|W_{6}\right|+\left|W_{7}\right|\left[\right.$ [2] allowing to take $W_{7}=0$. We find that with either $\hat{a}_{8} \neq 0$ or $\hat{a}_{6} \neq 0$ we can reproduce the histogrammed $|v|=0$ and $|v|=1$ distributions in Fig. $\emptyset$ (top) equally well. But only with $\hat{a}_{6} \neq 0$ can we reproduce the $|v|=2$ distribution, too, as shown in Fig. 4 (bottom left).

We can explain the drastically different effect of $W_{6}$ and $W_{8}$ on the analytic prediction for $|v|=2$ by noting that the $W_{6}$-term, in WRMT, corresponds to a Gaussian fluctuating mass, see Eq. (2.4). The $\delta$-function index peak of the continuum theory is therefore smeared into a Gaussian peak with an amplitude that increases with $|v| . W_{6}$, therefore, does not introduce a repulsion between eigenvalues. On the contrary, the $W_{8}$-term of $\mathrm{W} \chi \mathrm{PT}$ is included in the representation of the Dirac operator, Eq. (2.3), of WRMT, and hence induces an eigenvalue repulsion, as can be seen from the red curve in Fig. 田 (bottom left). This repulsion is seen for all sectors with $|v|>1$ [2]. It is thus useful to include eigenvalues from configurations with $|v|>1$ for a determination of the low-energy constants from fits to eigenvalue distributions.

We finally note that, with clover improvement the nonvanishing $\left|\hat{a}_{6}\right|$ is about a factor 3-4 

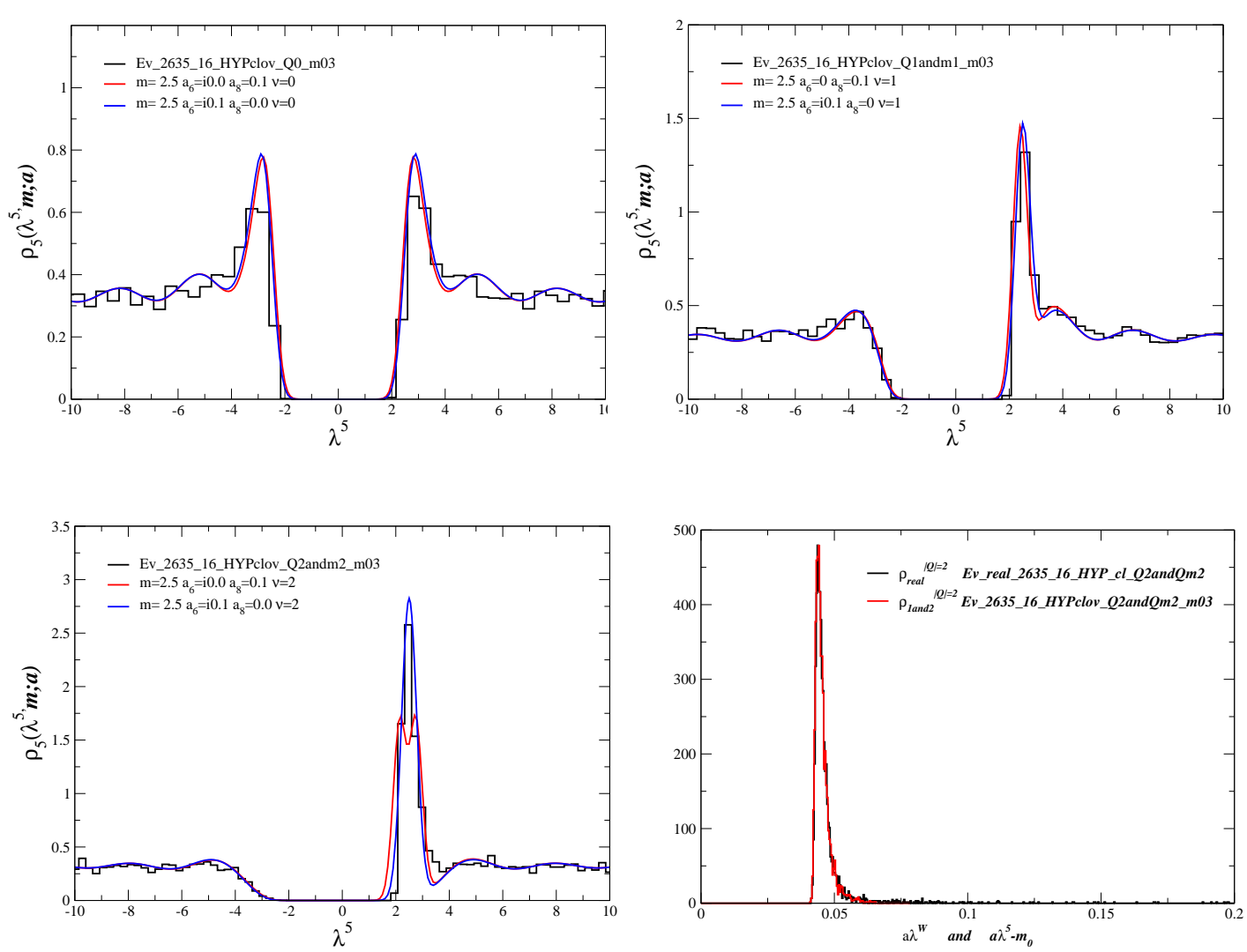

Figure 4: Comparison of the histogrammed clover-improved eigenvalue distributions with WRMT for ensemble A (top and bottom left). The $v=1$ distribution (top right) was used to obtain the WRMT parameters. The red curves are the WRMT predictions with $\hat{a}_{8} \neq 0$, the blue curves those with $\hat{a}_{6} \neq 0$. The bottom right plot shows a comparison of the distribution of the real eigenvalues with the first two positive eigenvalues of $D_{5}\left(m_{0}\right)$ shifted by the bare mass for the $v=2$ configurations.

smaller than the nonvanishing $\left|\hat{a}_{8}\right|$ without the improvement, both after one HYP smearing, illustrating again the quite dramatic effect of clover improvement on the $\mathscr{O}\left(a^{2}\right)$ low-energy constants.

\section{Conclusion}

We have presented numerical simulations, in the quenched case, of the low-lying eigenvalues of the Hermitian Wilson-Dirac operator, both with and without clover improvement to compare to predictions from $\varepsilon$-regime Wilson $\chi$ PT or, equivalently, Wilson RMT. We used the Iwasaki gauge action which suppresses dislocations and leads to a fairly unique index or topological charge. This is helpful, since the analytical predictions are made for sectors of fixed index. We found that our eigenvalue distributions agree well with the analytical predictions, and verified scaling with volume and (bare) mass.

We have also looked at the distribution of the real eigenvalues of the Wilson-Dirac operator, obtained from the spectral flow. We found a dramatic decrease of both the additive mass renormalization (the real eigenvalue peak is closer to zero) and the $\mathscr{O}\left(a^{2}\right)$ low-energy constants (the width of the distribution becomes narrower and more symmetric) with the clover improvement. 
Fits to the distribution of the low-lying eigenvalues of the Hermitian Wilson-Dirac operator allow determination of the low-energy constants of QCD including those that parameterize the $\mathscr{O}\left(a^{2}\right)$ lattice effects. However, distributions on configurations with $|v|>1$ are needed to disentangle the effects of $W_{8}$ from those of $W_{6}$ and $W_{7}$ when all $\left|\hat{a}_{j}\right|$ are small.

\section{Acknowledgments}

UMH thanks the organizers for a stimulating conference and the conveners of section A for the opportunity to present these results.

\section{References}

[1] S.R. Sharpe and R.L. Singleton, Phys. Rev. D 58 (1998) 074501 [arXiv:heplat/9804028];

G. Rupak and N. Shoresh, Phys. Rev. D 66 (2002) 054503 [arXiv:hep-lat/0201019];

O. Bär, G. Rupal and N. Shoresh, Phys. Rev. D 70 (2004) 034508 [arXiv:heplat/0306021];

S. Aoki, Phys. Rev. D 68 (2003) 054508 [arXiv:hep-lat/0306027];

S. Aoki and O. Bär, Phys. Rev. D 70 (2004) 116011 [arXiv:hep-lat/0409006];

A. Shindler, Phys. Lett. B672 (2009) 82 [arXiv:0812.2251];

O. Bär, S. Necco and S. Schaefer, JHEP 0903 (2009) 006 [arXiv:0812.2403].

[2] P.H. Damgaard, K. Splittorff and J.J.M. Verbaarschot, Phys. Rev. Lett. 105 (2010) 162002 [arXiv:1001.2937];

G. Akemann, P.H. Damgaard, K. Splittorff, and J.J.M. Verbaarschot, Phys. Rev. D 83 (2011) 085014 [arXiv:1012.0752];

G. Akemann and T. Nagao, JHEP 1110 (2011) 060 [arXiv:1108.3035];

M. Kieburg, J.J.M. Verbaarschot and S. Zafeiropoulos, Phys. Rev. Lett. 108 (2012) 022001 [arXiv:1109.0656].

[3] K. Splittorff, BoS (Lattice 2012) 018 [arxiv:1211.1803].

[4] P.H. Damgaard, U.M. Heller and K. Splittorff, Phys. Rev. D 85 (2012) 014505 [arXiv:1110.2851].

[5] A. Deuzeman, U. Wenger and J. Wuilloud, JHEP 1212 (2012) 109 [arXiv:1110.4002].

[6] P.H. Damgaard, U.M. Heller and K. Splittorff, Phys. Rev. D 86 (2012) 094502 [arXiv:1206.4786].

[7] R.G. Edwards, U.M. Heller and R. Narayanan Nucl. Phys. B 535 (1998) 403 [arXiv:hep-lat/9802016].

[8] R. Narayanan and H. Neuberger, Nucl. Phys. B 443 (1995) 305 [arXiv:hep-th/9411108];

H. Neuberger, Phys. Lett. B417 (1998) 141 [arXiv:hep-lat/9707022].

[9] Y. Iwasaki, Report No. UTHEP-118, 1983 [arXiv:1111.7054].

[10] S. Takeda et al. (CP-PACS Collaboration), Phys. Rev. D 70 (2004) 074510 [arXiv:hep-lat/0408010]; S. Necco, Nucl. Phys. B 683 (2004) 137 [arXiv:hep-lat/0309017].

[11] A. Hasenfratz and F. Knechtli, Phys. Rev. D 64 (2001) 034504 [arXiv:hep-lat/0103029].

[12] T.A. DeGrand, A. Hasenfratz and T.G. Kovacs, Nucl. Phys. B 505 (1997) 417 [arXiv:heplat/9705009].

[13] Y. Shamir, B. Svetitsky and E. Yurkovsky, Phys. Rev. D 83 (2011) 097502 [arXiv:1012.2819].

[14] M. Kieburg, K. Splittorff and J.J.M. Verbaarschot, Phys. Rev. D 85 (2012) 094011 [arXiv:1202.0620].

[15] M.T. Hansen and S. Sharpe, Phys. Rev. D 85 (2012) 014503 [arXiv:1111.2404];

Phys. Rev. D 85 (2012) 054504 [arXiv:1112.3998]. 